\title{
A Simple System \\ of Reaction-Diffusion Equations Describing Morphogenesis: Asymptotic Behavior $(*)$.
}

\author{
F. Rotme (Tübingen) - P. DE MotToni (Roma)
}

\begin{abstract}
Summary. - A system of reaction-diffusion equations with a single non-linear term is invesigated, such that the solutions remain bounded uniformly for infinite time, but the homogeneous equitibrium state is unstable. Using a Ljapunov functional and the compactness of the trajectories of the system, the solutions are proved to approach the equilibritum states. The stability character of the latter states is shown to be determined by a simpler selfadjoint problem.
\end{abstract}

\section{1. - Introduction.}

In his fundamental paper [16] TURING had shown that coupled reaction-diffusion equations may describe differentiation and formation of spatial patterns in biological systems. More precisely, by the establishment of concentration gradients of certain substances (morphogenes) in an assembly of cells, a "pre-pattern " is formed which triggers for instance cell differentiation [2]. More detailed models, which specify the chemical interaction, has been investigated by Auchuury and HeRschкowITzKadfmann [1, 7], as well as by Meinhardt and Gierer [10]. These models are quite complicated and difficult to treat by purely analytical tools. MAGINu [9] deals with a mathematically simpler model, where the equations are made as linear as possible, still retaining the qualitative features of Meinhardt's model. Here we shall be concerned with the analytical investigation of Maginu's equations, which in [9] were treated under special assumptions.

These equations describe the evolution of the concentrations $U \geqslant 0, V \geqslant 0$ of two morphogenes, called activator and inhibitor respectively. The activator is produced by autocatalysis and destroyed by the inhibitor. On the other hand, the activator produces the inhibitor, which in turn decays. All these processes are assumed to be linear, besides the autocatalysis, where saturation effects occur to prevent an uncontrolled growth. Then we may write the following system

$$
\left\{\begin{array}{c}
\frac{d U}{d t}=\varphi(U)-V \\
\varepsilon \frac{d V}{d t}=U-V+V_{i},
\end{array}\right.
$$

(*) Entrata in Redazione il 29 giugno 1978 .

10 - Annali di Matematica 
where three constants have been eliminated by appropriate normalization. Here $\nabla_{i} \geqslant 0$ describes a possible constant inhibitor production present even if both activator and inhibitor are absent (a similar effect for the activator can be incorporated in the definition of $\varphi$ ). The function $\varphi$ describes autocatalysis and saturation effects. We assume, besides $V_{i} \geqslant 0$, the following hypotheses:

i) there is only one intersection $(\tilde{U}, \tilde{V}) \tilde{U} \geqslant 0, \tilde{V} \geqslant 0$ between the isoclines $V=U+V_{i}$ and $V=\varphi(U)$, and

$$
\operatorname{sign}\left((U-\tilde{U})\left(\varphi(U-\tilde{U})-\left(U+V_{i}\right)\right)\right)<0,
$$

namely only one equilibrium solution for $\left(2_{0}\right)$ exists, and damping of the autocatalysis can only oceur for large activator concentrations: in particular, this means that $\varphi(0)-V_{i}>0$, namely the zero-point activator production in absence of activator is larger than that of the inhibitor.

ii) $\varphi\left(\varphi(0)-V_{i}\right) \leqslant 0$ : namely, saturation effects do occur, such that at activator concentration $\hat{U}=\varphi(\hat{\theta})-V_{i}$ the activator production is inhibited.

In terms of the phase-plane picture, this means that the system $\left(2_{0}\right)$ possesses, in the positive $(U, \nabla)$-quarterplane, the invariant rectangle $\mathcal{R}_{0}=\left[0, \varphi(0)-V_{i}\right] \times$ $\times[0, \varphi(0)]$ (see fig. 1 , where a typical sistuation is depicted).

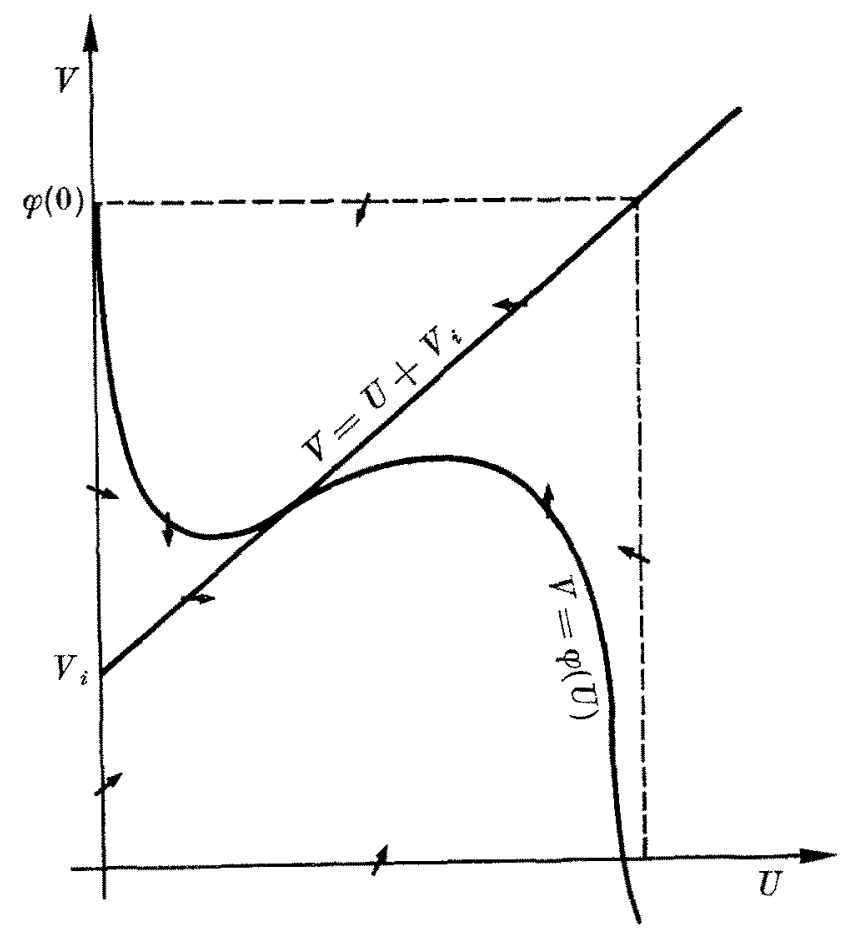

Figure 1 
In the following, we shall shift the origin of the coordinates to $(\tilde{U}, \tilde{V})$ : putting $u=U-\tilde{U}, v=V-\tilde{V}, f(u)=\varphi(u+\tilde{U})-\tilde{V}$, the equations $\left(2_{0}\right)$ read

$$
\left\{\begin{array}{l}
\frac{d u}{d t}=f(u)-v \\
\varepsilon \frac{d v}{d t}=u-v
\end{array}\right.
$$

In terms of the new quantities, the above assumptions read:

$$
\begin{gathered}
f(u)=u \quad \text { if and only if } u=0 ; \quad \operatorname{sign}(u(f(u)-u))<0 \\
f(f(-\tilde{U}))+\tilde{V} \leqslant 0 .
\end{gathered}
$$

An invariant rectangle is then given by

$$
\Re=[-\tilde{U}, f(-\widetilde{U})] \times[-\tilde{V}, f(-\tilde{U})]
$$

Adding diffusion terms, the system above reads (possibly after further normalization)

$$
\frac{\partial u}{\partial t}=\mu \Delta u+f(u)-v ; \quad \varepsilon \frac{\partial v}{\partial t}=\Delta v+u-v
$$

These equations should be supplemented by initial and boundary conditions. Like in Meinhardt's model, it turns out that the diffusion coefficient of the activator should be much smaller than that of the inhibitor (which is here normalized to one). Intuitively, this can be explained as follows: there will be sharp peaks in the activator concentration $u$, surrounded by much larger and shallower clouds of inhibitor, which prevent the activator peak to flow apart.

Our equations are very similar to the Fitzhugh-Nagumo equations describing the nerve axon conduction, as far as the reaction terms and the shape of $f(u t)$ are concerned; the main difference is that in such equations there is no diffusion of the inhibitor [4].

The present paper is organized as follows: in Section 2 we state the model and perform the classical stability analysis of the (trivial) homogeneous solution $u=0$, $v=0$. This shows the destabilizing effect of diffusion-which is one of the main discoveries of TURING [16]. In fact, whereas the trivial equilibrium $u=v=0$ is stable for the "space clamp" system (2). whenever $\varepsilon<1 / \lambda$, it becomes unstable if diffusion terms with different diffusion coefficients are introduced. In Section 3, using invariant rectangles [3] we shall prove the relative compactness of the trajectories of (1) (see [11], [13], [18] for similar techniques). Then making use of an appropriate Ljapunov functional and of a version of LaSalle's theorem [6], [8], we shall show that any solution of (1) approaches, as $t \rightarrow \infty$, the set of all equilibrium 
solutions of (1) (To show that the trajectories converge to a single equilibrium solution, it then sufficies to prove that the set of all equilibrium solutions is discrete). In Section 4 we shall show that the asymptotic stability character of the equilibrium solutions with respect to (1) for $\varepsilon \in(0,1]$ can be read off from the linear stability analysis of the "reduced" case $\varepsilon=0$, corresponding to infinitely fast reacting inhibitor. The detailed stability analysis of this case will be the object of a subsequent work [14].

\section{2. - Statement of the model and Turing's stability analysis.}

The (relative) concentrations $u$ and $v$ of activator and inhibitor are assumed to be functions of $(t, x) \in[0, \infty) \times \Omega$, where $\Omega$ is a bounded domain in $\boldsymbol{R}^{n}$. As explained in Sect. $1, u$ and $v$ will in general assume valnes of both signs. To get a well-posed problem, the model equations

$$
\left\{\begin{array}{l}
\frac{\partial u}{\partial t}=\mu \Delta u+f(u)-v \\
\varepsilon \frac{\partial v}{\partial t}=\Delta v+u-v \quad \text { for }(t, x) \in(0, \infty) \times \Omega,
\end{array}\right.
$$

must be supplemented by initial and boundary conditions. We shall consider (as Meinhardt does) the Neumann homogeneous conditions

$$
\frac{\partial u}{\partial n}=\frac{\partial v}{\partial n}=0 \quad \text { for }(t, x) \in(0, \infty) \times \partial \Omega,
$$

which express the requirement of zero flux across the boundary. Alternatively, we can consider Dirichlet homogeneous conditions

$$
u=v=0 \quad \text { for }(t, x) \in(0, \infty) \times \partial \Omega
$$

When $\Omega=[0, L]$ or $\Omega=\left[0, L_{1}\right] \times\left[0, L_{2}\right]$, one can think of removing the boundary of $\Omega$ : this amounts to imposing periodic boundary conditions, which, e.g. in the case $\Omega=[0, L]$, read:

$$
\begin{array}{ll}
u(t, 0)=\imath t(t, L) & u_{x}(t, 0)=u_{x}(t, L) \\
v(t, 0)=v(t, L) & v_{x}(t, 0)=v_{x}(t, L) \quad \text { for } t>0
\end{array}
$$

The initial conditions are

$$
\begin{aligned}
& u(0, x)=u_{0}(x) \\
& v(0, x)=v_{0}(x) \quad \text { for all } x ;
\end{aligned}
$$


in case $\varepsilon=0$, the initial conditions for $v$ should be dropped. Let us now state the assumptions about the domain $\Omega$ and the function $f$. Let $q \geqslant 0$ be an integer. The ease $q=0$ corresponds to the minimal differentiability assumption. For $q \geqslant 1$ one gets additional smoothness results.

$(\Omega q)$ Let the domain $\Omega$ be bounded and connected with smooth boundary: more precisely, assume there exist local representations of the boundary of the form

$$
x_{i}=r_{i}\left(x_{1} \ldots x_{i-1}, x_{i+1} \ldots x_{n}\right),
$$

where $D_{x}^{m} r_{i}$ are Hölder continuous for all $|m| \leqslant q+2$.

(f1) The function $f$ is locally Lipschitz continuous on $\boldsymbol{R}$, and differentiable for $u=0$ :

(f1q) $f$ has Hölder continuous derivatives up to order $q$.

Furtherly: With $\tilde{U}, \tilde{V}$, as introduced in sect. 1 , assume $(f 2),(f 3)$ to be valid.

In the following, it will be convenient to decompose the function $f$ into linear and higher order terms:

$$
f(u)=\lambda u-g(u),
$$

where $g(0)=g^{\prime}(0)=0$.

Let us now turn to the stability analysis of the trivial solution. To this end, consider first the limiting case $\varepsilon=0$. Thinking of $u$ as a known function, the second equation in (1)

$$
\Delta v+u-v=0
$$

together with the boundary condition for $v$, determines $v$ as a solution of a boundary value problem: in fact, $v$ can be expressed as

$$
v=B u,
$$

where the operator $B$ is bounded and continuous from the space $L^{p}(\Omega)$ to the Sobolev space $W^{2, p}(\Omega)$ for all $1 \leqslant p \leqslant \infty$, and from the space $C^{\alpha}(\Omega)$ to the space $C^{2+\alpha}(\Omega)$. As an operator from $L^{2}(\Omega)$ to $L^{2}(\Omega), B$ is a selfadjoint positive operator. Let $\left\{z_{p}(x)\right\}_{p \in N}$ the complete orthonormal system of eigenfunctions of the Laplacian on the domain $\Omega$ with boundary conditions $(1 a),(1 b)$ or $(1 c)$, and let $\left\{-k_{p}^{2}\right\}$ be the corresponding eigenvalues

$$
\Delta z_{p}+k_{p}^{2} z_{p}=0 \quad \text { on } \Omega ; p=1,2, \ldots
$$

The functions $z_{p}$ are of course eigenfunctions of $B$ as well:

$$
B z_{p}=\left(1+k_{p}^{2}\right)^{-1} z_{p} \quad \text { on } \Omega ; p=1,2, \ldots .
$$


It is then obvious that our model, in the limiting case $\varepsilon=0$, can be formulated as a single equation for $u$

$$
u_{t}=\mu \Delta u-B u+f(u)
$$

together with initial and boundary conditions for $u$.

The eigenvalue problem determining the stability is

$$
\mu \Delta w-B w+\lambda w=\alpha w \quad \text { on } \Omega
$$

(together with the appropriate boundary condition).

This problem has a pure point spectrum: namely the operator

$$
C=-\mu \Delta+B
$$

has the eigenfunctions $z_{p}$

$$
C z_{p}=\lambda_{p} z_{p}
$$

with eigenvalues

$$
\lambda_{p p}=\mu k_{p}^{2}+\left(1+k_{p}^{2}\right)^{-1}
$$

Hence the eigenvalues of $\left(6^{*}\right)$ are $\alpha=\lambda-\lambda_{p}$. Now, stability occurs if all eigenvalues $\alpha$ lie in the left half-plane: let $m \in \boldsymbol{N}$ an index for which $\lambda_{p}$ is minimal

$$
\lambda_{m}=\min \left\{\lambda_{p} ; p \in N\right\}
$$

Then the trivial equilibrium is stable if $\lambda<\lambda_{m}$ (and unstable if $\lambda>\lambda_{m}$ ). Since $\lambda_{m}>\min \left\{\mu x+(1+x)^{-1} ; x \geqslant 0\right\}=\sqrt{\mu}(2-\sqrt{\mu})>0$, the trivial equilibrium is always stable for $\lambda<\sqrt{\mu}(2-\sqrt{\mu})$. On the other hand, the "space clamp " system (2) reduces, if $\varepsilon=0$, to the single equation

$$
u_{t}=(\lambda-1) u+g(u)
$$

so that the trivial solution is stable whenever $\lambda<1$. This shows, in the case $\varepsilon=0$, the destabilizing effect of the diffusion: namely, for $\mu$ small enough, the trivial equilibrium solution is stable for the space clamp equation $\left(2^{*}\right)$ but unstable for the equation with diffusion if $\lambda_{m}>\lambda>1$.

So far we have considered the case $\varepsilon=0$. For $\varepsilon>0$, the situation is similar. The eigenvalue problem determining the stability is namely

$$
\begin{gathered}
\mu \Delta w+\lambda w-y=\alpha w \\
\Delta y+w-y=\varepsilon \alpha y \quad \text { in } \Omega,
\end{gathered}
$$


together with boundary conditions. As the operators arising in the left hand side all commute, only a pure point spectrum occurs: we may in fact take $w(x)=w_{p} z_{p}(x)$, and $y(x)=y_{p} z_{p}(x)$ (for $p \in \boldsymbol{N}$ ). Then (6) decomposes into simple matrix eigenvalue problems (one for each $p$ ):

$$
M_{p}\left(\begin{array}{l}
w_{p} \\
y_{p}
\end{array}\right)=\alpha\left(\begin{array}{l}
w_{p} \\
y_{p}
\end{array}\right)
$$

where

$$
M_{p}=-k_{p}^{2} D+M_{0}=-k_{p}^{2}\left(\begin{array}{cc}
\mu & 0 \\
0 & 1 / \varepsilon
\end{array}\right)+\left(\begin{array}{cc}
\lambda & -1 \\
1 / \varepsilon & -1 / \varepsilon
\end{array}\right) .
$$

The matrix $M_{0}$ determines the stability of the trivial solution of the space-clamp system (2): this solution is in fact stable if

$$
0 \leqslant \varepsilon<1 / \lambda
$$

and unstable if $\varepsilon>1 / \lambda$.

Choosing now $\varepsilon$ in order to satisfy (12), it turns out that det $M_{0}>0$ and trace $M_{0}<0$. Let us now investigate the stability of the full system, which is determined by the eigenvalues of $M_{p}$. Now, trace $M_{p}=-k_{p}^{2}($ trace $D)+$ trace $M_{0}$ which is strictly negative. Hence stability occurs if $\operatorname{det} M_{p}>0$. But a straightforward calculation yields

$$
\operatorname{det} M_{p}=-\left(1+k_{p}^{2}\right)\left(\lambda-\lambda_{p}\right) / \varepsilon,
$$

so that we have stability if $\lambda<\lambda_{p}$, for all $p$, (and instability if, for some $p, \lambda_{p}<\lambda$ ). We may therefore conclude:

Lемма 1. - Let (12) be satisfied (hence the irivial equilibrium solution is asymptotically stable for the space clamp system (2)). Then for the system with diffusion, the trivial equilibrium is stable if $\lambda<\lambda_{m}=\min _{p \in N}\left(\mu k_{p}^{2}+\left(1+k_{p}^{2}\right)^{-1}\right)$, and unstable if $\lambda>\lambda_{m}$.

Additional informations can be obtained about the eigenvalues of the matrix $M_{p}$ which cause the instability. In fact, instability occurs as det $M_{p}$ changes its sign (becoming negative), whereas trace $M_{p}$ keeps remaining negative. This means that one eigenvalue of $M_{p}$ is always negative, and that the essential eigenvalue causing the instability is real and positive. Thus under the restriction (12) the model cannot give rise to Hopf-type bifurcations, where purely imaginary eigenvalues occur at the onset of instability and give rise to periodic solutions. The Lyapunov functionals constructed in the next section will make sure that time-periodie oseillations cannot in fact occur at all. 


\section{3. - Existence, bounds and asymptotic behavior of the model equations.}

The existence of solutions of our model (1) follows from well-known results, we re-state here for the convenience of the reader:

THEOREVI 1. - Let the domain $\Omega$ satisfy the regularity assumption $(\Omega q)$ with $q=0$, let the fanction $f$ be globally Lipschitz continuous. Let the functions $u_{0}(\cdot)$ and $v_{0}(\cdot)$ be continuous. Then the differential equations (1), with initial conditions (1d) and boundary conditions (1a), (1b) or (1e) do have a unique solution for all time $t \geqslant 0$, which grows at most exponentially. If smoothness assumptions $(\Omega q)$ and $(f 1 q)$ are satisfied with $q>0$, then $u, v$ have Hölder continuous derivatives $D_{x}^{m} u, D_{x}^{m} v$ for $|m| \leqslant q+2$, and all $(t, x) \in(0, \infty) \times \bar{\Omega}$. In case $\varepsilon=0$, the problem can be formulated as equation $\left(1^{*}\right)$, with boundary and initial conditions for $u$ onty. Within these modifications, the same results hold true.

For the proof, see e.g. [4], and chap. III of [5].

The next step consists in removing the hypothesis of global Lipschitz continuity for $f$ we have used in Theorem 1, and at the same time to get time independent bounds for the solutions. To this end, we use the technique of the invariant rectangles (see CoNwAY and Syolder, [3]), which is a sharper version of the maximum principle, expounded as well in [17] (chap. IV, No. 32). By an invariant rectangle we mean a rectangle $\mathcal{R}$ in the phase plane of the space-clamp system (2), with sides parallel to the axes, such that the vector field defined by (2) does not point outside on the boundary of $R$. Recall now that if $f$ satisfies assumptions $(f 2),(f 3)$ then $\mathcal{R}=[-\tilde{U}, f(-\tilde{U})] \times[-\tilde{V}, f(-\tilde{U})]$ is an invariant rectangle, Then:

THEOREM 2. - Let the domain $\Omega$ satisfy the regularity assumption $(\Omega q)$ with $q=0$; let the function $f$ satisfy $(f 1),(f 2),(f 3)$. Suppose the initial data $u_{0}, v_{0}$ are continuous and $\left(u_{0}(x), v_{0}(x)\right) \in \mathcal{R}, \forall x \in \Omega$. Then a unique global solution of $(1)$ exists, bounded for all $t>0$, and such that it belongs to $R$ for all $(t, x) \in(0, \infty) \times \bar{\Omega}$ :

$$
(u(t, x), v(i, x)) \in \mathcal{R} ;(t, x) \in(0, \infty) \times \bar{\Omega} .
$$

In addition, for any $\delta>0$, the spatial derivatives $D_{x} u(t, x)$ and $D_{x} v(t, x)$ are uniformly bounded for all $(t, x) \in[\delta, \infty) \times \bar{\Omega}$. If the regularity assumptions $(\Omega q),(f 1 q)$ are satisfied with $q>0$, the solution is Hölder continuously differentiable up to order $q+2$, and for any $\delta>0$, the derivatives $D_{x}^{m} u(t, x)$ and $D_{x}^{m} v(t, x)$ are uniformly bounded for $(t, x) \in[\delta, \infty) \times \bar{\Omega}$, for all $|m| \leqslant q+1$. Corresponding results hold in the case $\varepsilon=0$.

Proor. - The methods of invariant rectangles is treated in full detail in [3] (see as well [11] for a similar application). Estimates for the derivatives are obtained by reformulating (1) as an integral equations system, and-using the smoothing 
properties of the fundamental solution of the heat equation $0=\Delta u-\partial u / \partial t$ in $\Omega$ (with the appropriate boundary conditions)-differentiating with respect to the space variables (see [11], [13], [18]).

Let us now construct a Ljapunov functional. The cases $\varepsilon=0$ and $\varepsilon>0$ should be treated separately. Let $F(u)=\int_{0}^{u} f\left(u^{\prime}\right) d u^{\prime}$ be a primitive of $f$.

LEMrar 2. - Let $\varepsilon$ be zero, and make the assumptions of Theorem 1 (or of Theorem 2) with $q=0$. Then the functional

$$
\mathcal{L}(u)=\int_{\Omega}\left\{\frac{1}{2} \mu|\operatorname{grad} u|^{2}+\frac{1}{2} u(B u)-F(u)\right\} d x
$$

has, along the solutions of $\left(1^{*}\right)$, the derivative

$$
d \mathcal{L} / d t=-\int_{\Omega} u_{t}^{2} d x
$$

In other words, the functional $\mathcal{L}$ is decreasing, and becomes stationary exactly on the equilibrium solutions.

The proof is a simple calculation.

The case $\varepsilon>0$ is slightly more involved: let $w$ be defined by

$$
0=\Delta v+w-v
$$

or, in terms of the operator $B$ introduced before:

$$
v=B w .
$$

LEMMA 3. - Let $\varepsilon>0$, let $f$ satisfy $(f 1 q)$ with $q \geqslant 4$, and, for some $\beta>1$,

$$
-\beta(\beta+2)<\varepsilon f^{\prime}<\beta(2-\beta)
$$

Assume the other hypotheses of Theorem 1 (or Theorem 2) with $q \geqslant 4$. Then the functional

$$
\mathcal{L}(u, v)=\int_{\Omega} d x\left\{\frac{1}{2} \varepsilon w_{i}(B w)_{t}+\frac{1}{2} \mu|\operatorname{grad} w|^{2}+\frac{1}{2} w(B w)-F(w)\right\}
$$

is decreasing along the solutions of (1): more precisely:

$$
d \mathscr{L} / d t \leqslant-K_{\lambda} \int_{\Omega} d x\left\{v_{t}^{2}+\left(\Delta v_{t}\right)^{2}\right\}
$$

for some positive constant $K_{1}$. In addition, $\mathcal{L}(u(t), v(t))$ becomes stationary if and only if $(u, v)$ is an equilibrium solution for (1). 
Proof. - We differentiate the second equation in (1)

$$
\varepsilon v_{t}=u-w
$$

(reeall that $v=B w$ )

with respect to the time $t$ :

$$
\begin{aligned}
\varepsilon v_{t t} & =u_{t}-w_{t}=\mu \Delta u+f(u)-v-v_{t}+\Delta v_{t} \\
& =\mu \Delta w+f(w)-B w+\mu \Delta(u-w)+f(u)-f(w)-v_{t}+\Delta v_{t} .
\end{aligned}
$$

Using the relations (18) and the mean value theorem, we obtain

$$
\varepsilon(B w)_{i t}-\mu \Delta w-f(w)+B w=\left(-1+\varepsilon f^{\prime}(\theta)\right) v_{t}+(1+\mu \varepsilon) \Delta v_{t},
$$

$\theta$ being some value in the interval $(u, w)$. By multiplying both sides with $w_{i}=v_{t}-\Delta v_{t}$ and integrating over $\Omega$, it follows that

$$
\left.d \mathcal{L} / d t=\int_{\Omega}\left\{\left(-1+\varepsilon f^{\prime}(\theta)\right) v_{t}+(1+\mu \varepsilon) \Delta v_{t}\right)\left(v_{t}-\Delta v_{t}\right)\right\} d x
$$

The equation $w=u-\varepsilon v_{t}$ implies that also $w$ satisfies the same boundary conditions as $u$ and $v$. This fact can be used in partial integrations, as well as the selfadjointness of $B$. Because of the assumed estimate for $f^{\prime}$, the integrand above defines a negative definite bilinear form, whence (17) follows, together with the last claim of the Lemma.

Notice that in (16) $w$ and $w_{t}$ can be expressed in terms of $u, v$, so that $\mathcal{L}$ is actually a functional of $u, v$.

Theorem 2 together with Lemmata 2 and 3 can now be used to study the asymptotic behavior of the solutions of $(1),\left(1^{*}\right)$ by means of the LaSalle's theorem $[6],[8]$. To this end, it is convenient to consider (1) and (1*) as equations of the motion in a suitable Banach space $X$ (of continuous functions on $\Omega$ ). The trajectories of (1), (1*) are then defined in an obvious way. To any trajectory we associate the respective (positive) $\omega$-limit set $A_{x}^{+}$:

$\Lambda_{X}^{+}=\left\{w \in X\right.$; there is a sequence $t_{n} \nearrow \infty$ such that $w\left(t_{n}\right)$ converges to $w$ in $\left.X\right\}$.

The following Lemma states some basic properties of the $\omega$-limit sets (independent of any Ljapunov function):

LEMma 4. - Assume that the solutions of problem (1) (or (1*)) depend continuously on the initial data in the norm of $X$. Assume furtherly that there exists a $\delta>0$ such that the "final part " of the trajectory $\left\{w(t) \in X ; w(t)\right.$ is solution of $(1)$ or $\left.(1)^{*} ; t \geqslant \delta\right\}$ ¿is relatively compact in $X$. Then

a) Any limit set $\Lambda_{x}^{+}$is nonvoid, compact and connected; 
b) The trajectory approaches its own limit set in the norm of $X$ :

$$
\lim _{t \rightarrow \infty} \operatorname{dist}_{x}\left(w(t), \Lambda_{X}^{+}\right)=0 ;
$$

c) Any w-limit set is invariant: new trajectories which start at some point in $\Lambda_{x}^{+}$ remain in $A_{x}^{+}$for all times $t>0$.

The proof of this lemma is standard (see e.g. [6]). To apply LaSalle's theorem, the Ljapunov functionals has to be continuous on the Banach space $X$ : this determines our choice of $X$. Again, the cases $\varepsilon=0$ and $\varepsilon>0$ should be treated separately. For $\varepsilon=0$, the function $u$ obeys $(1 \tau)$ : in this case, we take

$$
X=C^{1}(\bar{\Omega})
$$

endowed with the standard (maximum) norm.

For $\varepsilon>0$, the appropriate choice is a space of couple of functions $(u, v)$, namely

$$
X=C^{4}(\bar{\Omega}) \oplus C^{4}(\bar{\Omega})
$$

with the usual norm. Then we are able to prove

Theorem 3. - Case $(a)$ : assume $\varepsilon=0$. Consider the parabotic equation (1*) with boundary conditions (1a), (1b) or (1c) and initial condition (1d) (for the component $u$ only). Assume $(\Omega q)$ with $q \geqslant 1$ and let the function $f$ satisfy $(f 1),(f 2),(f 3)$ and be Hölder continuously differentiable for $u \in[-\widetilde{U}, f(-\tilde{U})]$. Assume that the initial function $u_{0}$ is continnous and satisfies $u_{0}(x) \in[-\tilde{U}, f(-\tilde{U})]$ for all $x \in \Omega$.

Case $(b)$ : assume $\varepsilon>0$. Consider the parabolic system (1) with boundary conditions $(1 a),(1 b),(1 c)$ and initial conditions $(1 d)$. Assume $(\Omega q)$ with $q \geqslant 4$, and let the function $f$ satisfy $(f 1),(f 1 q)$ with $q \geqslant 4,(f 1)$ and $(f 3)$. Let $\varepsilon>0$ be such that

$$
-\beta(2+\beta)<\varepsilon f^{\prime}<\beta(2-\beta)
$$

Assume that the initial functions $u_{0}, v_{0}$ are continuous, and $\left(u_{0}(x), v_{0}(x)\right) \in \mathcal{R}, \forall x \in \Omega$. Defining $X$ as above, in both cases the following results hold true:

1) The w-limit set of any trajectory of $\left(1^{*}\right),(1)$ consists of equilibrium solutions of $\left(1^{*}\right),(1)$, respectively $\left({ }^{1}\right)$.

2) If all equitibrium solutions of (1) are isolated in $X$, then any solution of (1) converges to an equilibrium solution of (1) in the norm of $X$.

( $)$ In fact, the equilibrium solutions for $(1 *)$ and for (1) are essentially the same. 
REMARK. - It is not diffieult to prove that the equilibrium solutions are isolated whenever the stability operator $A=\mu \Delta-B+f^{\prime}(\hat{u})$ does not have the eigenvalue zero. Notice that, because of the last claim of Lemma 3, time-periodic oseillations cannot occur.

Proof. - By Theorem 2, the final part of the trajectories is relatively compact in $X$, and Lemmata 2, 3 apply. Proceeding like in [8], we observe that, $\mathfrak{E}$ being decreasing along the trajectories and bounded below, the limes

$$
\lim _{t \rightarrow \infty} \mathfrak{L}(w(t))=\mathfrak{L}_{\infty}>-\infty
$$

exists $\left(w(t)\right.$ denoting the solution of $\left(1^{*}\right)$ or of $\left.(1)\right)$. If $w\left(t_{n}\right) \rightarrow \hat{w}$ in the corresponding $\omega$-limit set, using the continuity of $\mathcal{L}$ it is easily seen that $\mathcal{L}_{\infty}=\mathfrak{L}(\hat{w})$ and that $\mathcal{L}$ is constant on the $\omega$-limit set of any trajectory: in other words, the $\omega$-limit sets consists of stationary points of $\mathcal{L}$, which, by Lemma 2 (respectively, Lemma 3) are equilibrium solutions of $\left(1^{*}\right)$ (respectively, (1)). This proves the first claim.

To prove the second one, observe that, if all equilibrium solutions of (1) are isolated in $X$, then item 1 of Lemma 4 implies that the $\omega$-limit sets consist of just one equilibrium solution, and the claim follows by Lemma 4,2 ).

REMARK. - In the case $\Omega \subseteq \boldsymbol{R}^{1}$, the conditions $(f 2),(f 3)$ can be replaced by

$$
F(u) \leqslant \Pi_{2} \quad \text { for all } u \in \boldsymbol{R} .
$$

In fact, this condition and the boundedness of $\mathcal{L}(u)$ imply that $u$ is bounded in $H^{1}(\Omega)$ and hence, by the Sobolev estimates, in $O(\bar{\Omega})$ as well.

\section{4. - Comparison of stability of equilibrium solutions for $\varepsilon=0$ and $\varepsilon>0$.}

Clearly, there is a one-to-one correspondence between the equilibrium solutions $(\hat{u}, \hat{v})$ for $(1)$ and the equilibrium solutions $\tilde{u}$ for $\left(1^{*}\right)$, which is established by putting $\hat{u}=\tilde{u}, \hat{v}=B \hat{u}$; however, linear stability analysis leads in the two cases to different eigenvalue problems.

From now on, let us assume $f$ to be continuously differentiable; then the stability of the equilibrium solution $(\hat{u}, \hat{v})$ is governed by the eigenvalue problem

$$
\mu \Delta u-B u+f^{\prime}(\hat{u}) u=\alpha u
$$

when $\varepsilon=0$, and by the eigenvalue problem

$$
\begin{aligned}
& \mu \Delta u+f^{\prime}(\hat{u})-v=\alpha(\varepsilon) u \\
& \Delta v+u-v=\alpha(\varepsilon) v
\end{aligned}
$$


when $\varepsilon>0$ (of course, (19*) and (19) should be supplemented by the appropriate boundary conditions). Let us now introduce the Banach space $Y$

$$
\begin{aligned}
& Y=O(\bar{\Omega}) \text { in case of boundary conditions }(1 a) \text { or }(1 c) \\
& Y=C_{0}(\bar{\Omega}) \text { in case of boundary conditions }(1 b)\left(^{2}\right)
\end{aligned}
$$

endowed with the "supremum" norm. Accordingly, we shall define $X=Y \oplus Y$. Problems (1) and $\left(1^{*}\right)$ will then be considered as ordinary differential equations in these spaces, whereas (19) and $\left(19^{*}\right)$ will be interpreted as stationary equations in such spaces. To formulate these equations in a rigorous way, let us define, for any equilibrium solution $(\hat{u}, \hat{v})$ the linear operator

$$
A=\mu \Delta-B+f^{\prime}(\hat{u}),
$$

with domain $D(A)$ :

$$
\begin{aligned}
D(A)= & \{u \in C(\bar{\Omega}) ; \Delta u \in C(\bar{\Omega}) ; u \text { satisfies the boundary condition }\} \\
& \text { in case of boundary conditions }(1 a),(1 c) ; \\
D(A)= & \left\{u \in C_{0}(\bar{\Omega}) ; \Delta u \in C(\bar{\Omega})\right\} \text { in case of boundary condition }(1 b) .
\end{aligned}
$$

With these definitions, $D(A)$ is in any case a dense domain in $Y$; hence $A$ is a densely defined closable operator on $Y\left({ }^{3}\right)$. Therefore we may define the densely defined, closable operator on $X$

$$
\mathfrak{X}^{\varepsilon}=\left(\begin{array}{lc}
\mu \Delta+f^{\prime}(\hat{u}) & -1 \\
1 / \varepsilon & 1 / \varepsilon(\Delta-1)
\end{array}\right)
$$

(the domain $D\left(\mathfrak{Q}^{\varepsilon}\right)$ being defined in an obvious way). The "stability equations " $\left(19^{*}\right)$ and (19) now read

$$
\begin{gathered}
A u=\alpha u \\
\mathfrak{H}^{\varepsilon}\left(\begin{array}{l}
u \\
v
\end{array}\right)=\alpha(\varepsilon)\left(\begin{array}{l}
u \\
v
\end{array}\right) .
\end{gathered}
$$

As usual, if $T$ is a linear operator on the Banach space $Z$, its resolvent and spectrum will be denoted by $\varrho_{z}(T), \sigma_{z}(T)$, respectively.

The following theorem shows that the linear stability or instability as read off from $(19),\left(19^{*}\right)$, are intimately related. In fact, we can prove

(2) We define $C_{0}(\bar{\Omega})$ as $\{u \in C(\bar{\Omega}), u=0$ on $\partial \Omega\}$.

(3) Notice that $A$ extends to a selfdjoint operator on $L^{2}(\Omega)$. 
Lemina 5. $-S$ Suppose $\sup \sigma_{Y}(A)=\alpha_{0}<0$. Then for $\varepsilon \in(0,1]$ there exist $\delta>0$ and $\theta \in(0, \pi / 2)$ such that the resolvent set $\varrho_{X}\left(\mathfrak{A}^{\varepsilon}\right)$ contains the translated sector $S_{x / 2+\theta}-\delta$, where $S_{\beta}=\{z \in C ;|\arg z|<\beta\}$. Furtheron, there exists, a constant $M(\delta, \theta)$ such that

$$
\left\|\left(\mathfrak{Q}^{s}-z\right)^{-1}\right\| \leqslant \frac{M(\delta, \theta)}{|z+\delta|}, \quad \text { for all } z \in \mathcal{S}_{\pi / 2+\theta}-\delta
$$

On the other hand, if $\sup \sigma_{Y}(A)>0$, then $\sup \operatorname{Re} \sigma_{X}(\mathfrak{A} \varepsilon)>0$.

As a consequence, observe that if $\sup \sigma_{Y}(A)<0$, then the operator $\mathfrak{A}^{\varepsilon}$ generates on $X$ an analytic semigroup of strictly negative type [15]. Then it is easy to prove the following

THEOREM 4. - If $\hat{u}$ is a linearly asymptotically stable solution of (1*) in the sense that $\sup \sigma_{Y}(A)<0$, then for all $\varepsilon \in[0,1]$ the equilibrium solution of $(1)$ given by $(\hat{u}, \hat{v})$ (where $\hat{v}=B \hat{u}$ ) is asymptotically stable. On the other hand, if $\hat{u}$ is a linearly unstable solution of (11) in the sense that $\sup \sigma_{Y}(A)>0$, then, for any $\varepsilon>0$, the corresponding equilibrium solution $(\hat{u}, \hat{v})$ of $(1)$ is unstable.

The proof of this theorem relies on Lemma 5 and, on the fact that "linear asymptotic stability implies asymptotic stability", namely the generalization to Banach spaces of the classical Ljapunov-Perron result on linearized stability: a key role is here played by the fact that the linearized operator generates a (strongly continuous) semigroup of strictly negative type [12].

Proof of Lemma 5. - Suppose $\sup \sigma_{Y}(A)=\alpha_{0}<0$. Consider the resolvent equation for $\mathfrak{A} \varepsilon$ :

$$
\left(\mathfrak{Q}^{\varepsilon}-v\right)\left(\begin{array}{l}
u \\
v
\end{array}\right)=\left(\begin{array}{l}
\varphi \\
\psi
\end{array}\right)
$$

or, explicitly:

$$
\begin{gathered}
\mu \Delta u+f^{\prime}(\hat{u}) u-v-v u=\varphi \\
\Delta v+u-v-\varepsilon v v=\varepsilon \psi
\end{gathered}
$$

The second equation can be solved for $v$ whenever

$$
v \in C-(-\infty,-1 / \varepsilon]
$$

Then the operator

$$
B(v)=(-\Delta+1+\varepsilon v)^{-1}
$$


is well-defined and continuous from $L^{2}(\Omega)$ to $H^{2}(\Omega)$ and from $C(\bar{\Omega})$ to $C^{1+\alpha}(\bar{\Omega}){ }^{(4)}$. Substituting for $v$ the expression $B(v)(u-\varepsilon \psi)$ into the first equation of $(21)$, we get:

$$
A(v) u=\varphi-\varepsilon B(\nu) \psi,
$$

where

$$
A(\nu)=\mu \Delta+f^{\prime}(\hat{u})-v-B(\nu)
$$

For $\nu \in C-(-\infty,-1 / \varepsilon]$, the operators $A(v)$ form a selfadjoint analytic family, and have all a pure point spectrum. In particular, this implies that $A(\nu)$ is invertible if and only if zero is not an eigenvalue of $A(v)$. In such case, $A(v)^{-1}$ is continous from $L^{2}$ to $H^{2}$ and from $C$ to $C^{1+\alpha}$. Hence, if $v \in C-[-\infty,-1 / \varepsilon]$ and zero is not an eigenvalue of $A(\nu)$, then $\mathfrak{A}^{\varepsilon}-\vartheta$ is invertible and given by

$$
\left(\mathfrak{A}^{\varepsilon}-v\right)^{-1}=\left(\begin{array}{cl}
A(v)^{-1} & -\varepsilon A(v)^{-1} B(v) \\
B(v) A(v)^{-1} & -\varepsilon B(v)\left[A(v)^{-1} B(v)+1\right]
\end{array}\right)
$$

which is a continuous operator from $L^{2} \oplus L^{2}$ to $H^{2} \oplus H^{2}$ and from $C \oplus C$ to $C^{1+\alpha} \oplus C^{1+\alpha}$. It remains to be shown that, if $v$ is in a suitable translated sector $S_{\pi / 2 ; \theta}-\delta$, then zero is not an eigenvalue of $A(v)$. To this end, let $j(v)$ be an eigenvalue of $A(v)$ and $w(v)$ a corresponding normalized eigenfunction. Denoting by $($,$) the inner product in$ $L^{2}(\Omega)$, we obtain:

$$
j(v)=(A(v) w(v), w(v))
$$

Using the resolvent identity, we get $A(\nu)=A-\nu+\varepsilon v B(v) B$.

Let us now split $v$ into real and imaginary parts: $v=v_{1}+i v_{2}$. Analogously, put $j=j_{1}+i j_{2}$; using the fact that $\varepsilon v B(\nu) B=B(\nu) B(\bar{v})\left[\varepsilon v+\varepsilon^{2}|v|^{2} B\right]$, we get:

$$
\begin{aligned}
& j_{1}\left(\nu_{1}, \nu_{2}\right)=(A w, w)-\nu_{1}+\varepsilon v_{1}(b(v), b(\nu))+\varepsilon^{2}|v|^{2}(B b(v), b(v)) \\
& j_{2}\left(\nu_{1}, v_{2}\right)=-v_{2}+\varepsilon v_{2}(b(v), b(v))
\end{aligned}
$$

where $b(\nu)=B(\nu) w(\nu)$.

Using the semidefiniteness of $\Delta$ and the expansion into eigenfunctions of $\Delta$, one shows that

$$
0<(B b(\nu), b(v)) \leqslant(b(\nu), b(v)) \leqslant\left(\left(1+\varepsilon v_{1}\right)^{2}+\left(\varepsilon v_{2}\right)\right)^{-1}
$$

(4) In the course of this proof we consider, for definiteness, only the case of boundary conditions $(1 a),(1 c)$. 
Suppose now $\nu \in \sigma_{X}(\mathfrak{K} \varepsilon)$ : then zero is an eigenvalue of $A(v)$. This implies first $j_{2}=0$, hence:

$$
\nu_{2}=0, \quad \text { or } \quad\left(1+\varepsilon \nu_{1}\right)^{2}+\left(\varepsilon v_{2}\right)^{2} \leqslant \varepsilon .
$$

Secondly, $j_{1}=0$ as well, so that

$$
0 \leqslant \sup \sigma_{Y}(A)-\gamma_{1}+\frac{\varepsilon^{2}|v|^{2}+\varepsilon \max (0,1)}{\left(1+\varepsilon v_{1}\right)^{2}+\left(\varepsilon v_{2}\right)^{2}} .
$$

If $\sup \sigma_{X}(A)=-\left|\alpha_{0}\right|<0$ and $0<\varepsilon<\left(1-\left|\alpha_{0}\right|\left(^{-1}\right.\right.$ for $\left|\alpha_{0}\right|<1$ and $0<\varepsilon$ (arbitrary) for $\left|\alpha_{0}\right| \geqslant 1$, the relations (23) and (24) imply that $y$ lies in the strip

$$
\left\{v_{1}+i v_{2} ; v_{1} \leqslant \eta<0,\left|v_{2}\right| \leqslant \varepsilon^{-\frac{1}{2}}\right\} \text {, }
$$

for some positive $\eta$. Then the spectrum of $\mathfrak{A}^{\varepsilon}$ lies in this strip, so that the resolvent contains a sector $S_{t / 2+\theta}-\delta$ with the required properties. (We also see that the condition $\varepsilon \leqslant 1$ can be relaxed).

Consider now the case $\sup \sigma_{Y}(A)=\alpha_{0}>0$. Let $w(0)$ be the eigenvector belonging to the maximum eigenvalue $j(0)$ of $A=A(0)$. Since $A(v)$ is a selfadjoint analytic family of operators with pure point spectrum, there exist eigenvalues $j(v)$ with eigenvector $w(v)$ such that $j(0)=\alpha_{0}, j(\nu)$ is differentiable, and $(w(v), w(v))=1$.

Now the estimate $j_{1}\left(\nu_{1}, 0\right) \leqslant \alpha_{0}-v_{1}+\left(\varepsilon \nu_{1}\right) /\left(1+\varepsilon v_{1}\right)$ implies $\lim _{\nu_{1} \rightarrow \infty} j_{1}\left(v_{1}, 0\right)=-\infty$. On the other hand, $j_{1}(0,0)=\alpha_{0}>0$. As $j_{2}\left(\nu_{1}, 0\right)=0$ (for any $\nu_{1}$ ), there is a $\tilde{\nu}_{1}>0$ such that $j_{1}\left(\tilde{v}_{1}, 0\right)=j_{2}(\tilde{v}, 0)=0$.

This implies that $\left(\tilde{v}_{1}, 0\right)$ belongs to $\sigma_{X}(\mathfrak{Q} \varepsilon)$.

To complete the proof, we have to establish the required estimate for $\left\|(\mathfrak{A} \varepsilon-v)^{-1}\right\|$ in the case $\sup \sigma_{Y}(A)=\alpha_{0}<0$. First, observe that $-\Delta$ generates an analytic semigroup on $X[15]$, hence

$$
\|B(v)\| \leqslant N_{1} /(|v+1 / \varepsilon|) \quad \text { for } \nu \in \mathbb{S}_{\pi / 2+\theta}-\delta,
$$

and $A$ generates an analytic semigroup of strictly negative type; hence, by possibly redefining $\theta \in(0, \pi / 2)$ and $\delta>0$ :

Now,

$$
\left\|(A-v)^{-1}\right\| \leqslant N_{2} /\left(\mid v-\alpha_{0}\right) \quad \text { for } v \in \mathbb{S}_{\pi / 2+\theta}-\delta .
$$

$$
A(v)=(A-v)\left\{I+(A-v)^{-1} \varepsilon v B(v) B\right\}
$$

where the operator in curly brackets is invertible for $v \in S_{\pi / 2}-\delta$ if

$$
\left\|(A-v)^{-1} \varepsilon v B(\nu) B\right\| \leqslant \frac{N_{1} N_{2}\|B\| \varepsilon v}{|\nu+1 / \varepsilon| \cdot\left|v-\alpha_{0}\right|} \leqslant r<1 .
$$

This is certainly satisfied, say, with $r=\frac{1}{2}$, whenever $v$ lies outside the disc $D=\{z \in C ;|z+\delta| \leqslant R\}, R$ being suitably chosen. With this choice, (25) implies

$$
\left\|A(v)^{-1}\right\| \leqslant N_{3} /(|\nu+\delta|) \quad \text { for } \nu \in\left(S_{\pi / 2+\theta}-\delta\right)-D .
$$


On the other hand, because of the analyticity of $A(\nu)$, a similar estimate holds in $\left(S_{\pi / 2+\theta}-\delta\right) \cap D$. These estimates for $\left\|A(\nu)^{-1}\right\|$ and $\|B(\nu)\|$ imply the required estimate for $\left\|\left(\mathfrak{A}^{\varepsilon}-v\right)^{-1}\right\|\left({ }^{5}\right)$.

\section{REFERENCES}

[1] J. F. G. AUCHMUTY - G. NICOLIS, Bifurcation analysis of nonlinear reaction-diffusion equations. I: Evolution equations and the steady state solutions, Boll. Math. Biol., 37 (1975), pp. 323-365.

[2] A. Babloyantz - J. Hiernaux, Models for cell differentiation, Bull. Math. Biol., 37 (1975), pp. 633-657.

[3] E. D. Conway - J. A. SMoLLER, A comparison technique for system of reaction-diffusion equations, Comm. in Part. Differ. Equations, 2 (7) (1977), pp. 679-698.

[4] J. Evans - S. Shenk, Solutions to axon equations, Biophys. Journ., 10 (1970), pp. 1050-1101.

[5] A. Friedman, Partial Differential Equations of Parabolic Type, Prentice-Hall, Englewood Cliffs, N.J., 1964.

[6] J. HaLe, Dynamical systems and stability, J. Math. Anal. Appl., 26 (1969), pp. 39-59.

[7] M. HERsChKowItZ-KAUFMANN, Bifurcation analysis of nonlinear reaction-diffusion equations. II: Steady state solutions and comparison with numerical simulations, Bull. Math. Biol., 37 (1975), pp. 589-635.

[8] J. P. LASALLE, An invariance principle in the theory of stability, Intern. Symp. on Differential Equations and Dynamical Systems, ed. by J. K. HALE and J. P. LASALLE, Academic Press, New York, 1967; The stability of dynamical systems, SIAM Regional Conference Series in Applied Mathem., no. 25, SIAM, Philadelphia, 1977.

[9] K. MAGINU, Reaction-diffusion equations describing morphogenesis. I: Waveform stability of stationary wave solutions in a one-dimensional model, Math. Biosci., 27 (1975), pp. 17-98.

[10] H. MEInhaRDT, A model of pattern formation in insect embryogenesis, J. Cell. Sci., 23 (1977), pp. 117-139.

[11] P. DE MOTTONI - F. RothE, Convergence to the constant homogeneous equilibrium state in generalized Volterra-Lotka systems with diffusion, SIAM, J. Appl. Math. (in print).

[12] P. DE MotToni - A. Tesei, Asymptotic stability results for a systems of quasilinear parabolic equations, Applicable Analysis, 7 (1979), pp. 7-21.

[13] F. Rothe, Convergence to the equilibrium state in the Volterra-Lotka diffusion equations, Journ. Math. Biol., 3 (1976), pp. 319-324.

[14] F. RoTHE, A simple system of reaction-diffusion equations describing morphogenesis. Existence and stability of non-homogeneous equilibrium states, preprint (1978).

[15] H. B. StewarT, Generation of analytic semigroups, Trans. Amer. Math. Soc., 199 (1974), pp. 141-162.

[16] A. M. Tuking, The chemical basis of morphogenesis, Phil. Trans. Roy. Soc., B 237 (1952), pp. 37-72.

[17] W. WALTER, Differential and integral inequalities, Springer-Verlag, Berlin - Heidelberg New York, 1970.

[18] S. Wichtams - P. CHOw, Nonlinear reaction-diffusion models for interacting populations, Journ. Math. Anal. Appl., 62 (1978), pp. 157-169.

(5) For a similar argument, see ref. [12].

11 - Annali di Matematica 